
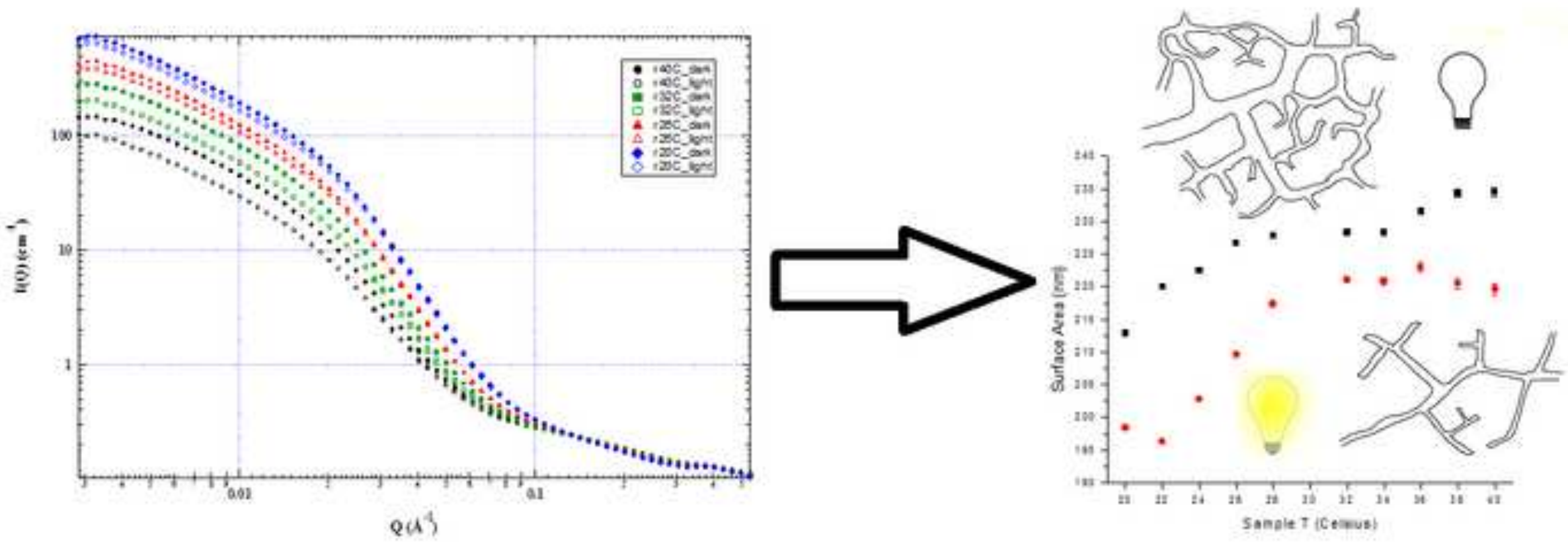


\title{
Illumination Alters the Structure of Gels Formed from the Model Optoelectronic Material P3HT
}

Brian Morgan ${ }^{1}$, and Mark D. Dadmun ${ }^{1,2}$

\begin{abstract}
Studying the gelation process of conjugated optoelectronic polymers has often been employed as a means of better understanding the final morphology and assembly in active layers of organic electronic devices due to the correlation between the experimentally observed sol-gel transition and many common solution based fabrication techniques. The nature of the percolated network structures formed through the molecular assembly that occurs during this gelation directly affects device performance in conjugated polymer based active layers. Thus, precise knowledge of the evolution of structures during gelation provides crucial information that is needed to rationally improve device performance by directing the assembly during processing. Additionally, observing the effects of environmental factors such as ambient light exposure upon the gelation process will direct efforts towards improving universally overlooked facets of the typical fabrication procedure. Thus, we have conducted a series of ultra small angle and small angle neutron scattering experiments to probe the temperature-driven gelation process of the conjugated photoactive polymer poly(3-hexylthiophene-2,5-diyl) (P3HT) in both the presence and absence of white light. Analysis of the resultant scattering data shows that the gelation
\end{abstract}


process consists of the creation and steady growth of cylindrical aggregates formed by the agglomeration of free chain P3HT. Furthermore, clear differences in the gel structure and assembly between illuminated and non-illuminated gels are observed across multiple length scales, pointing towards an optically-induced variation in the gelation process. Our results indicate that simple white light exposure sharply retards the growth of conjugated polymer microstructures, which clearly suggests that ignoring illumination conditions throughout organic electronic fabrication processes risks producing inconsistent and non-reproducible active layer architectures and ultimately endangers dependable device performance.

\section{Introduction}

Fabrication of electronic devices that incorporate novel conjugated optoelectronic polymers has led to an exciting field of active layer design resulting in lightweight, cost effective, and application tunable electronics. Ranging from photovoltaics, to field effect transistors, to organic LEDs; these devices capitalize on the semiconducting nature of the polymer to effectively transport the respective charge carriers throughout the active layer. Designing these polymer active layers to best facilitate charge carrier creation, movement, and collection has been a focal point of research in the area for decades. ${ }^{1,2,3,4,5}$ However much room for improvement remains as device efficiency and stability weaknesses persist. ${ }^{6}$ To best optimize crucial active layer performance parameters such as exciton dissociation efficiencies, the donor and acceptor layers must be engineered in such a way that interfacial area is maximized to ensure effective charge transport pathways within range of typical exciton dissociation lengths in these materials $(\sim 10 \mathrm{~nm}){ }^{7}$ This being the case, careful morphological control of the active layer structure becomes vital, as these optoelectronic properties are well known to be highly dependent on local composition and chain conformation. ${ }^{8}$ 
Due to the complexity of the systems of interest, effectively elucidating the exact structures of the constructs present within conjugated polymer active layers is a nontrivial undertaking. Thus, much of the work in the field has revolved around altering fabrication techniques to achieve morphologies with improved performance rather than directly engineering the materials themselves to form optimal morphological structures. As a result, processes which occur naturally during the typical fabrication process are of great interest, and gaining insight into how best to control them to achieve optimal structures becomes key.

One of the most crucial production processes associated with commonly used optoelectronic conjugated polymers is polymer gelation, the process by which a 3-D polymer rich network percolates in a phase that lies somewhere between liquid and solid. ${ }^{9}$ Knowledge of the gelation process assists in the development of certain electronic inks and printed films; processes which seek to avoid large-scale gelation due to its tendency to form blockages and inhomogeneities within the desired system. ${ }^{10}$ But more importantly for broader purposes, studying the gelation process allows insight into the pathway of aggregate formation in optoelectronic polymers, as it acts as a form of polymeric self-assembly conducive to the formation of stable structure networks that are effective charge carriers. ${ }^{11}$ Gaining insight into the specific details of the self-assembly process can allow substantial structural control of the final film through variations in polymer concentration, solvent identity, and gelation impetus; amongst other experimental variables. ${ }^{12,13}$ One of the more common manifestations of these effects occurs during typical organic electronics device fabrication techniques such as spincoating. Over the relatively brief timescale of spin-coating, a polymer film is created through the quick removal of solvent via fluid motion and evaporation. ${ }^{14}$ Due to the rapid loss of the favorable solvent environment, an accelerated formation of aggregates occurs until kinetically 
frozen by sufficient solvent withdrawal. The gelation mechanisms (such as nanofibril formation and macro-aggregation) observed in solution systems drive this process as well, albeit in modified form on a different timescale. Thus, a fundamental understanding of solution gelation mechanisms carries over to the formation of bulk films by specific processing methods and consequently can aid the design of tunable electronics fabrication methods in order to produce desired morphologies and architectures. ${ }^{15,16,17}$

There exists a wide range of proposed pathways through which some of the more common conjugated polymers aggregate during the gelation process including percolation ${ }^{18}$ and phase separation. ${ }^{19}$ Specifically for poly(3-hexylthiophene-2,5-diyl), an optoelectronic benchmark polymer, a multi-step physical gelation process is often assumed, as illustrated in Figure 1. During the initial stages of applied system stress, such as decreased temperature or solvent quality, strong inter- and intra-chain $\pi$-stacking interactions cause aggregation of individual P3HT chains into small bundles known as nanowhiskers or nanofibrils. ${ }^{20}$ These nanostructures themselves further aggregate into clusters to begin the onset of gelation, ultimately forming a viscoelastic 3-D network, which percolates throughout the sample. Though these rough physical outlines of the $\mathrm{P} 3 \mathrm{HT}$ gelation process are generally well known, the exact quantitative nature of the structures formed as they evolve throughout the stimuli ramp is mostly unclear. In this work, we present a quantitative and qualitative analysis of structure factors of the gelling of P3HT in a favorable solvent monitored closely step-by-step throughout a temperature ramp, and these scattering data are analyzed to quantitatively monitor the crucial structural parameters as they evolve. 

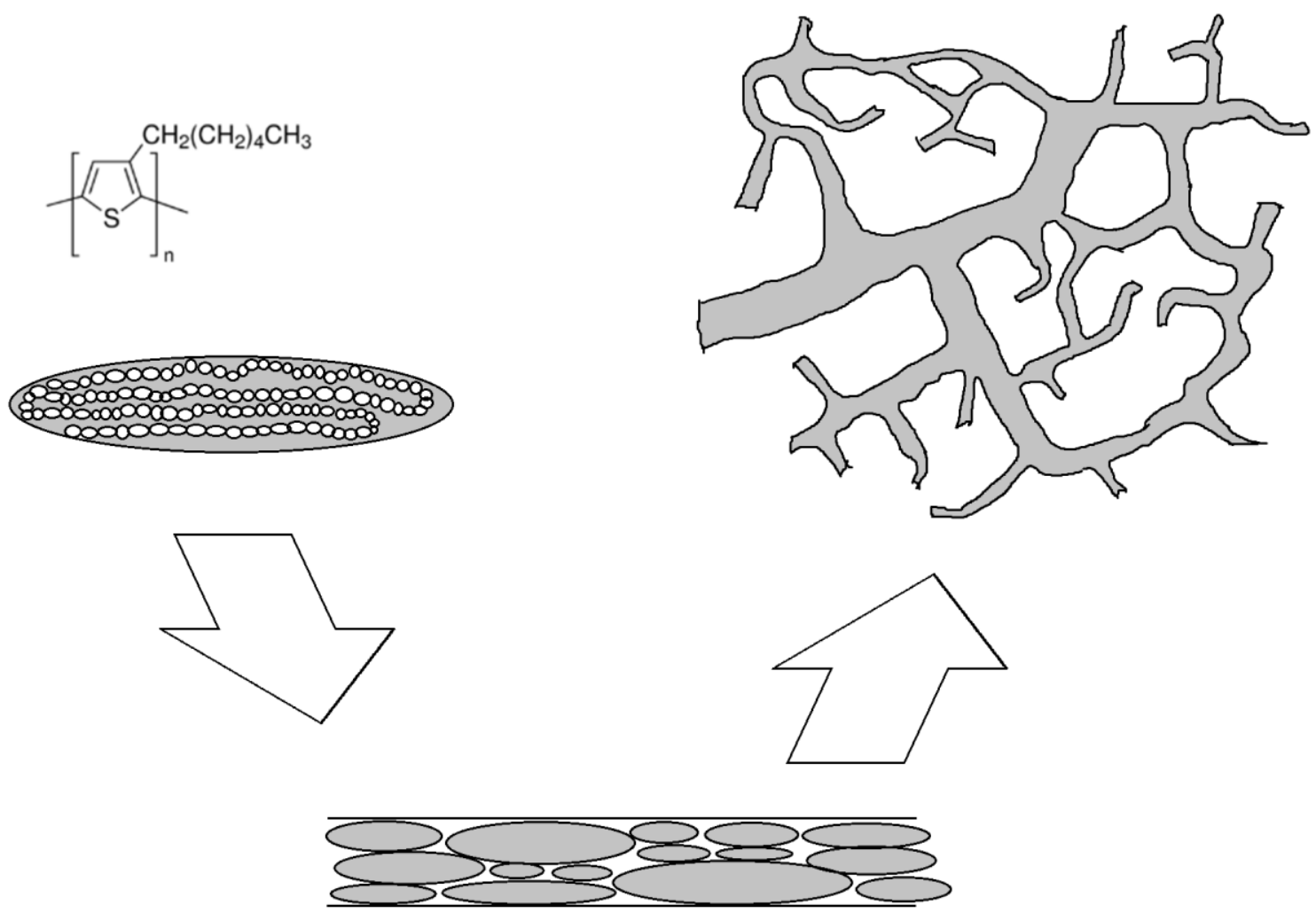

Figure 1: Schematic depiction of the assembly of P3HT in solution to form a self-supporting gel. The length scales increase from left to right, depicting free chains that assemble into nanofibrils, which further assemble into a larger gel.

Though much attention across the field of optoelectronic polymers has been focused on optimizing morphological environments in order to induce favorable device efficiencies, the question of whether or not the process which provides the driving force behind the device mechanisms, i.e. the formation of excitons, may itself be influencing polymer chain conformation has remained somewhat of an afterthought. Current preliminary experiments hint towards the validity of this postulate, potentially opening the door to a wide range of opportunities to control polymer morphology before and during device processing, through 
selective illumination of sensitive active layers. For instance, simulations of PPV oligomers predicted a planarization of the polymer backbone due to the presence of the delocalized charges, which are self trapped over six repeat units. ${ }^{21}$ Similarly, an investigation by Reiter et. al. through a series of dewetting experiments of conjugated polymer thin films demonstrated a clear and reversible change in viscosity between polymer films annealed under white light versus in total darkness, a phenomenon tentatively attributed to exciton-driven rigidification of the polymer backbone. ${ }^{22}$ Additional studies have shown a range of deviations in physical behavior of optoelectronic polymers due to the presence of excitons. ${ }^{23,24}$ Building off of these works, our group has looked to establish a clear link between the presence of light induced excited species and alterations in polymer chain conformation. Several experiments using small angle neutron scattering that probe the dilute solution behavior of conjugated polymers in marginal solvents with and without white light illumination have established that real, significant, and reproducible changes in polymer conformation occur upon light exposure. ${ }^{25}$ These results show a clear decrease in structural parameters, including $\mathrm{R}_{\mathrm{g}}$ and Kuhn length upon exposure to light, along with a decline of the second virial coefficient. We interpret these results to indicate that the presence of excitons on the polymer backbone decreases the solvent quality of the system and leads to a contraction of the chain that significantly decreases the size of the polymer chain, particularly in marginal solvent conditions. Thus, in addition to monitoring structural changes throughout gelation, this work seeks to expand our understanding to the impact of the presence of light on the self-assembly of conjugated polymer chains in concentrated solution by monitoring the effect of in-situ white light illumination on the structures that form during the gelation process. Moreover, the degree to which this illumination influences the structural 
growth of the gel network is also monitored, as these characteristics will ultimately impact the foundation of subsequent active layer device morphologies.

\section{Experimental}

Gelation samples were created using poly(3-hexylthiophene-2,5-diyl) purchased from Rieke Metals with a regioregularity $>98 \%$ and a molecular weight $<50,000$, per the manufacturer. The polymer material was dissolved in deuterated 1,2 ortho-dichlorobenzene (ODCB) to give a sample consisting of $17 \%$ P3HT by weight $(275 \mathrm{mg} / \mathrm{mL})$. Samples were elevated to $70^{\circ} \mathrm{C}$ to ensure complete dispersion of the polymer in the solvent. All experimental sequences were conducted using a temperature ramp which employed thermostat heating controls to move in set temperature increments through gelation to its completion at room temperature. Starting at $40^{\circ} \mathrm{C}$, scattering data were collected at temperatures every $2^{\circ} \mathrm{C}$ down to $20^{\circ} \mathrm{C}$, with a 15 minute thermal equilibration allowed between each data collection period. Following a gelation sequence, the samples were again returned to $70^{\circ} \mathrm{C}$ and maintained there until re-use as part of a subsequent run with a change in illumination conditions.

Small angle neutron scattering (SANS) experiments were conducted at the CG-2 GPSANS beamline of the High Flux Isotope Reactor (HFIR) at Oak Ridge National Laboratory. Scattering was collected at a single sample-detector distance using a neutron wavelength of $6 \AA$, allowing a $Q$ range ranging from 0.003 to $0.5 \AA^{-1}$, where $Q=4 \pi / \lambda \sin (\theta / 2)$ with $\lambda$ being the neutron wavelength and $\theta$ the scattering angle. Raw data was reduced using SPICE ORNL reduction protocol macros in Igor Pro. ${ }^{26}$ Samples were mounted in $2 \mathrm{~mm}$ wide glass banjo cells holding approximately $0.4 \mathrm{~g}$ of P3HT solution. 
Ultra small angle neutron scattering (USANS) experiments were performed at the National Institute of Standards and Technology in Gaithersburg, Maryland on the BT-5 beamline. Using six buffers and an initial neutron wavelength of $2.4 \AA$ allowed for collection of scattering data down to $3.5 \times 10^{-5} \AA^{-1} \cdot{ }^{27}$ Raw data was reduced using the NIST NCNR reduction and desmearing macro suite in Igor Pro. Curve fitting analysis of both the USANS and SANS data was performed using NCNR analysis macros in Igor Pro as well as the DANSE SasView software project.

To achieve proper light/dark environments, a consistent methodology was adhered to. "Dark" trials were conducted by wrapping the sample cells in $0.1 \mathrm{~mm}$ aluminum foil, effectively blocking out all incident photons while remaining transparent to neutrons. Removing the foil and illuminating the sample in-situ at a $5 \mathrm{~cm}$ distance throughout the data collection runs with an optical illuminator consisting of a 125 watt halogen bulb providing 50,000 lux created the "light" conditions for the appropriate trial runs. This intensity is verified with a calibrated light meter at the sample cell itself. This offers illumination conditions significantly more intense than the simple ambient light conditions found under standard laboratory conditions, a typical illumination environment which generally amounts to approximately 1,000 lux of light intensity. Analyzing a solution of $1 \mathrm{wt} \%$ polystyrene under the above stated "light" experimental conditions provides a control sample and verifies that the changes in scattering data are due to the presence of the illumination, and not an experimental artifact. Representative data for this control experiment is available in a previous publication, ${ }^{25}$ confirming that both the "light" and "dark" curves overlap well throughout the entire $Q$ range, as expected. As a result, the methodology utilized in these experiments to collect neutron scattering of conjugated polymer solutions under 
illuminated and non-illuminated conditions appears valid and free from error associated with thermal effects or scattering artifacts that are due to the presence of aluminum.

\section{Results}

Small angle neutron scattering data was collected for the $17 \mathrm{wt} \%$ P3HT sample at each temperature along a thermal ramp starting at $70^{\circ} \mathrm{C}$ to ensure full polymer dispersion in the solvent, and then continuing from $40^{\circ} \mathrm{C}$ to $20^{\circ} \mathrm{C}$ in $2^{\circ} \mathrm{C}$ increments. This polymer concentration was arrived at after a series of cloud point experiments (available in the Supplemental Information) which indicated that $17 \mathrm{wt} \%$ offered the ideal experimentally feasible system to simulate the high-concentration environment present during solution based casting procedures upon rapid solvent evaporation and displacement. The process was conducted first without ambient light, and immediately repeated under illumination, employing identical thermal histories for both trials. The resultant data curves are shown in Figure 2, wherein filled data points correspond to experimental trials conducted in the dark, and open data points indicate trials performed under white light illumination. Overall the samples produce powerful scattering curves indicative of large aggregates formed at these high concentration conditions. Increased scattering intensity is seen as temperature decreases, an intuitive trend considering the rapid growth of the strongly scattering P3HT aggregates as gelation progresses. Large differences in absolute intensity are observed in the low Q regions between light and dark samples for all temperatures, with the dark samples consistently exhibiting much stronger scattering. These differences in absolute intensity range from 5-20\% for most samples, up to an increase of over $40 \%$ for the lowest $\mathrm{Q}$ region of the higher temperature samples. However for $\mathrm{Q}>0.1 \AA^{-1}$ all intensity differences have dissipated and universal overlap for all temperatures is observed, indicating little change in local chain structure of the samples throughout the gelation process. 


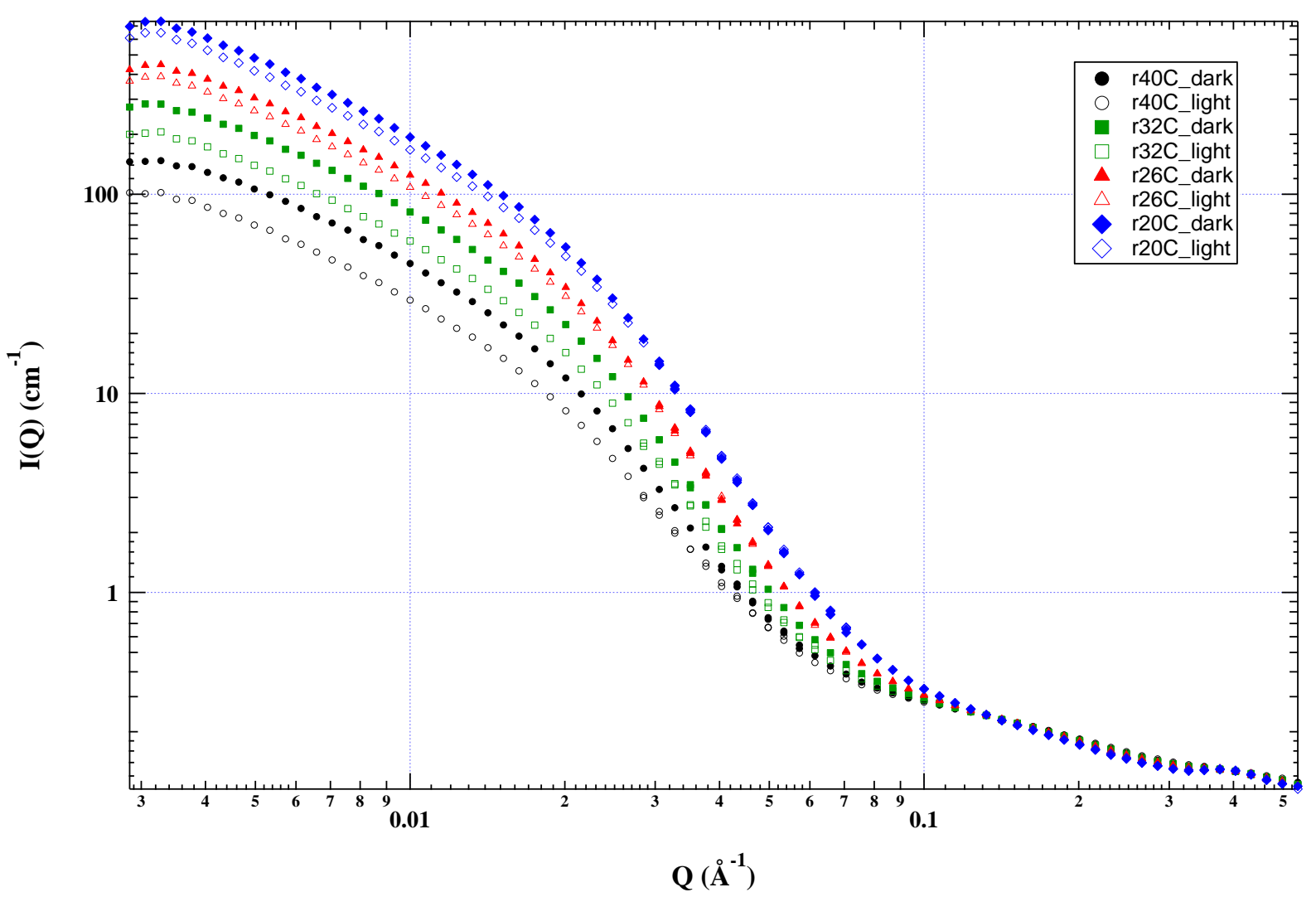

Figure 2: SANS of $17 \mathrm{wt} \%$ P3HT gelation under illumination (open symbols) and in the dark (closed symbols) through a $70-20^{\circ} \mathrm{C}$ temperature ramp. Color denotes example individual temperatures between $40^{\circ}$ and $20^{\circ} \mathrm{C}$

The complexity of the hierarchical structures that exist during the P3HT gelation process requires a multiscale model to properly fit the data. P3HT nanofibril aggregates are modeled using an elliptical cylinder or parallelepiped form factor which assumes an oval or rectangular aggregate cross section, respectively. ${ }^{28,29,30}$ Fitting to this model allows extraction of twodimensional parameters related to the surface area of the nanofibril face, the scattering length density of the aggregate, which is proportional to its composition, and a scale factor, which is proportional to the volume fraction of aggregates. In order to account for the presence of "free" P3HT chains not yet aggregated in solution, a polymer excluded volume model is included via linear addition. This allows the structural size and shape of the individual un-aggregated chains 
to be monitored throughout gelation by monitoring its radius of gyration and Porod exponent. The combination model approach has been shown to accurately fit the P3HT system under similar gelation conditions over a wide Q range encompassing all relevant SANS data. ${ }^{31}$ The $^{2}$ total combined model is described by:

$$
I(q)=\phi_{P 3 H T} \phi_{E C M}\left(\Delta \rho_{E C M}\right)^{2} P_{E C M}(q)+\phi_{P 3 H T}\left(1-\phi_{E C M}\right)\left(\Delta \rho_{P E V}\right)^{2} P_{P E V}(q)
$$

where $\phi_{P 3 H T}$ describes the total volume fraction of P3HT in the solution, $\phi_{E C M}$ is the volume fraction of aggregated P3HT present and modeled as an elliptical cylinder model, $P_{P E V}$ is the free chain excluded volume form factor for P3HT, $P_{E C M}$ describes the elliptical cylinder form factor for the aggregates, and $\Delta \rho_{E C M}$ and $\Delta \rho_{P E V}$ are the scattering length density contrast between P3HT aggregates and the solvent and between the free P3HT chains and the solvent, respectively. Figure 3 shows an example fit of the data to the combined ECM/PEV model for a given single temperature. Data fit well across the entire Q range using this model for the range of temperatures studied, including the crossover region between models. Through a fitting methodology based on systematically holding known parameters constant, a variety of structural parameters of the gelling sample are then extracted in both illuminated and dark environments. 


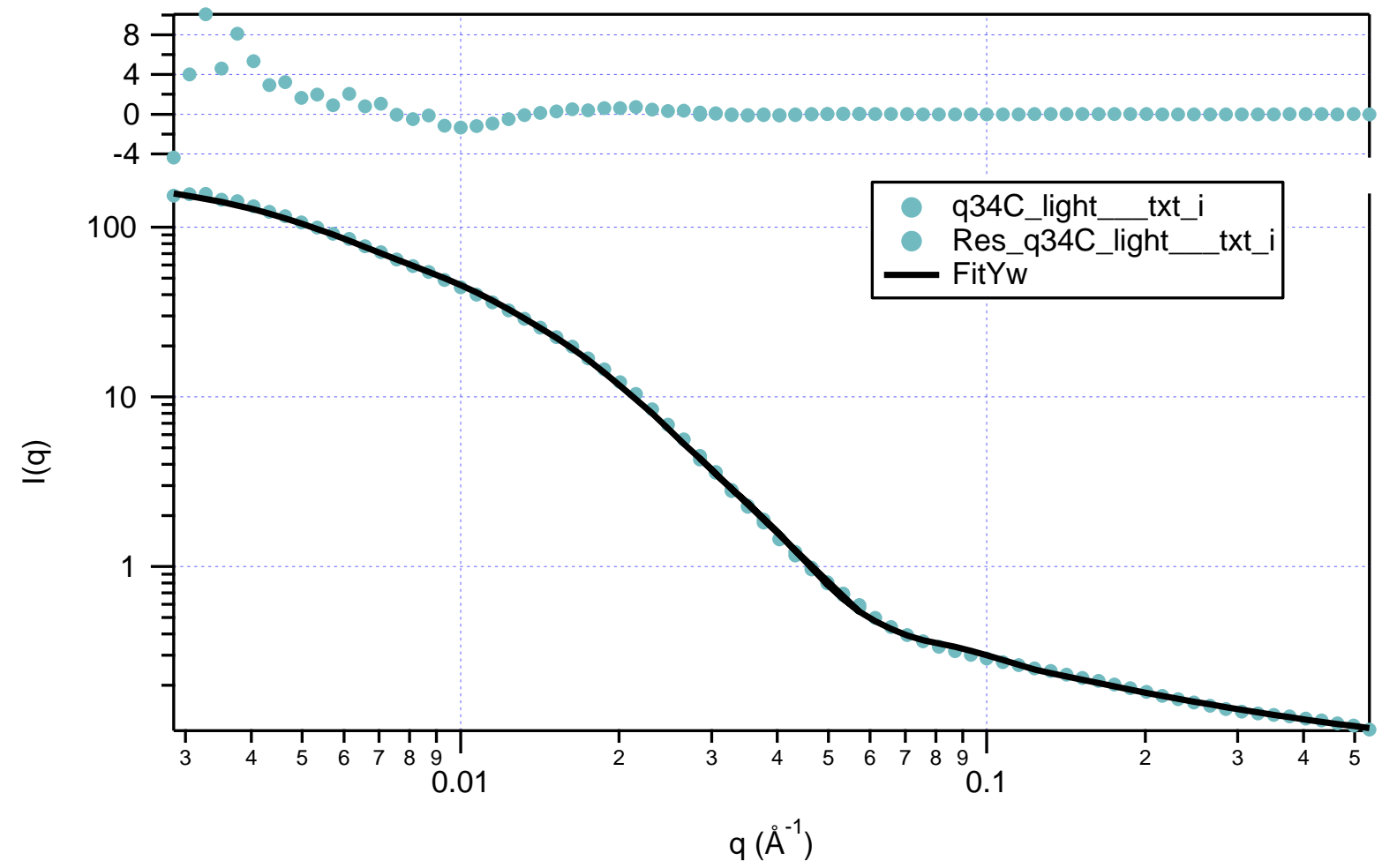

Figure 3: Combination Model (Elliptical Cylinder + Polymer Excluded Volume) fit to $17 \mathrm{wt} \%$ $\mathrm{P} 3 \mathrm{HT}$ gelation curve in $\mathrm{d}-\mathrm{ODCB}$ at $34^{\circ} \mathrm{C}$ in the presence of white light illumination.

Figures 4-7 illustrate the evolution of select parameters during the gelation process in both the light and dark. Figure 4 gives values for the cross sectional area of the P3HT nanofibrils described by the elliptical cylinder model, essentially describing the cross-sectional area of the nanofibril faces. Our data show this parameter is consistently smaller in the light than in the dark. Figure 5 displays the elliptical cylinder model scale factor, which is directly proportional to the amount of P3HT that exists in the aggregated nanofibrils, as opposed to existing in a free chain form. This value remains consistently larger in the dark than in the light throughout gelation. Use of the polymer excluded volume model gives the radius of gyration for free chains, and is shown in Figure 6, with the illuminated chains appearing larger than the non-illuminated chains, especially at higher temperatures. Finally, Figure 7 reports the Porod exponent values obtained 
from the excluded volume model for the free chains, a parameter related to the conformation of the polymer chain, with a value of 1 indicating a classic rigid rod while a value of 2 denotes a Gaussian coil. As gelation progresses, all values increase from a rigid rod-like state towards a more Gaussian conformation, with the illuminated sample values consistently somewhat smaller throughout. As with the raw data, these quantitative analyses show that there exist clear differences between light and dark samples in all structural parameters through the gelation process.

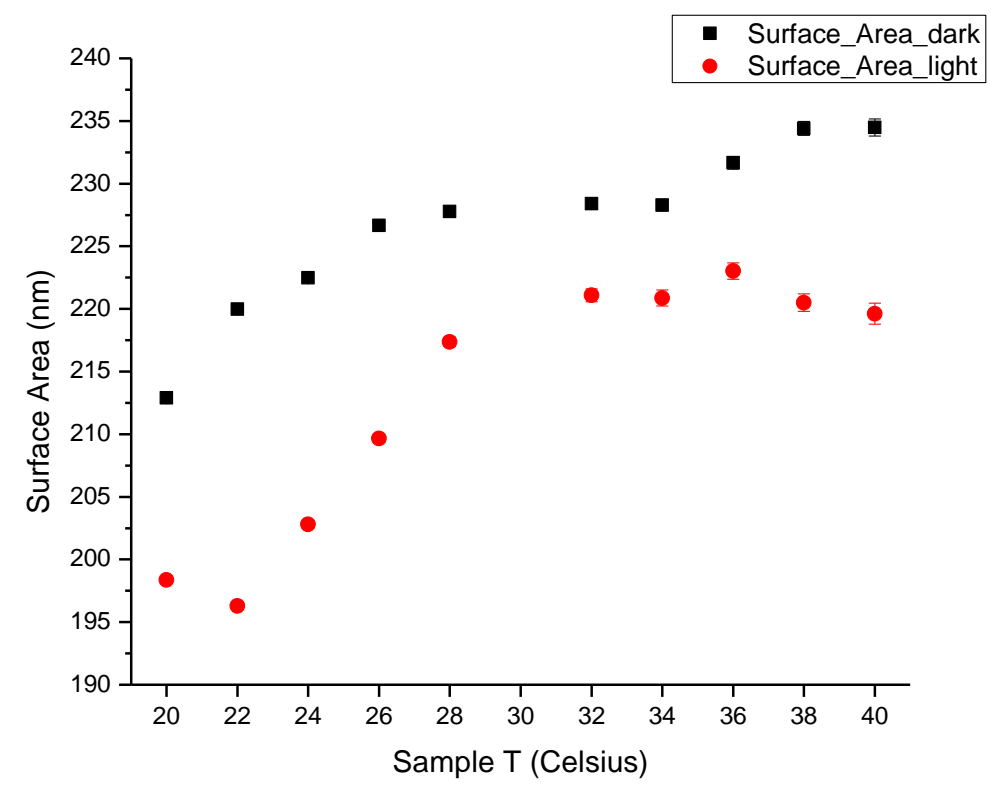

Figure 4: Surface area of the nanofibril face, i.e the cross-section of nanoscale P3HT fibers. This plot shows that the P3HT fibers are consistently larger in the dark than in the light. 


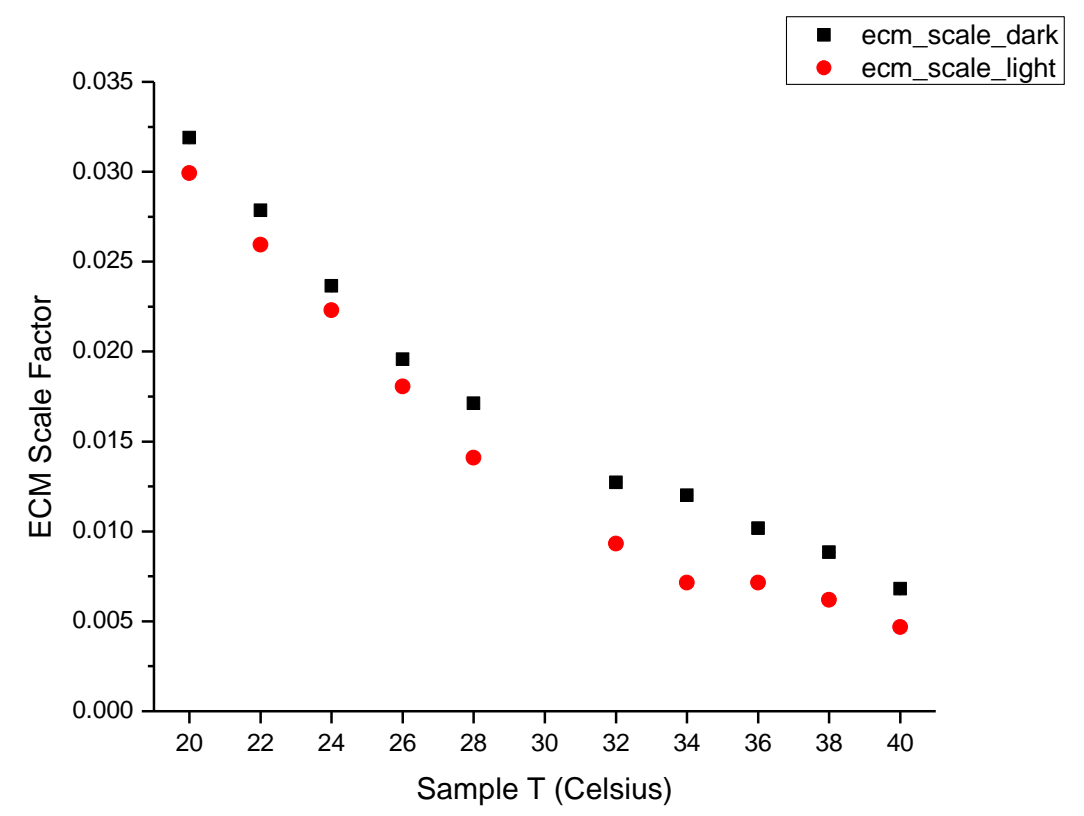

Figure 5: Scale factor obtained from the elliptical cylinder model portion of the fit, a parameter that is directly proportional to the amount of P3HT aggregated in nanofibrils. This data show that the amount of aggregated P3HT remains larger in the dark environment throughout gelation.

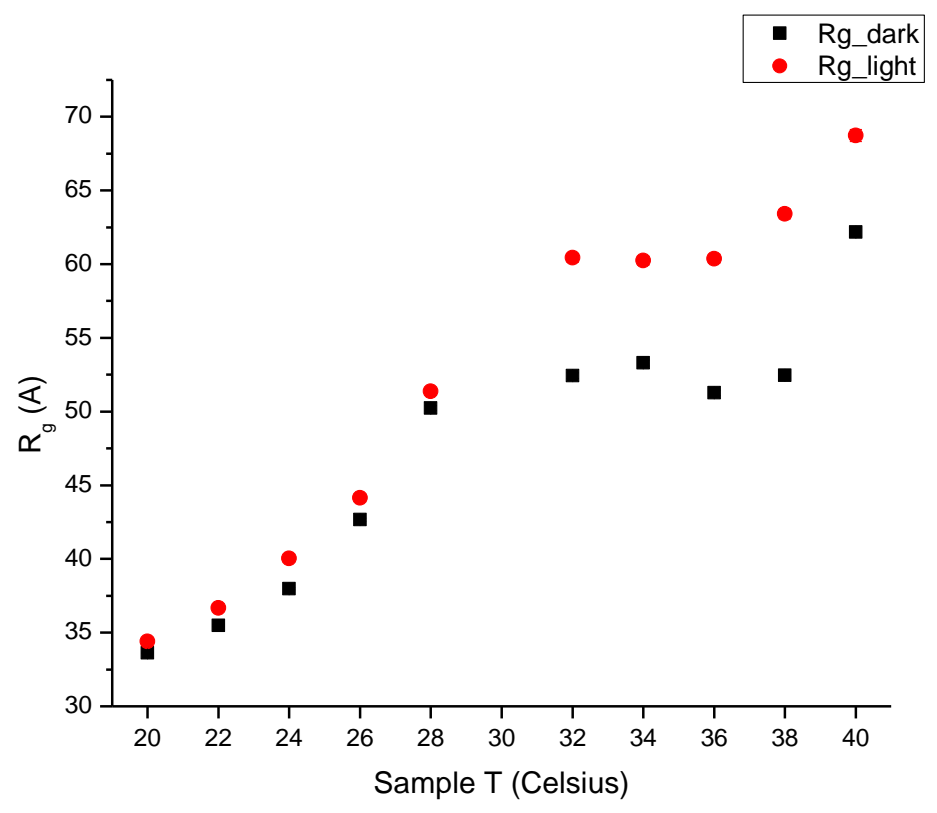

Figure 6: Radius of gyration of P3HT obtained from the excluded volume model, which monitors the size of free P3HT not aggregated in nanofibrils. This structural feature shows that the free chains are larger in the light than the dark, especially at higher temperatures. 


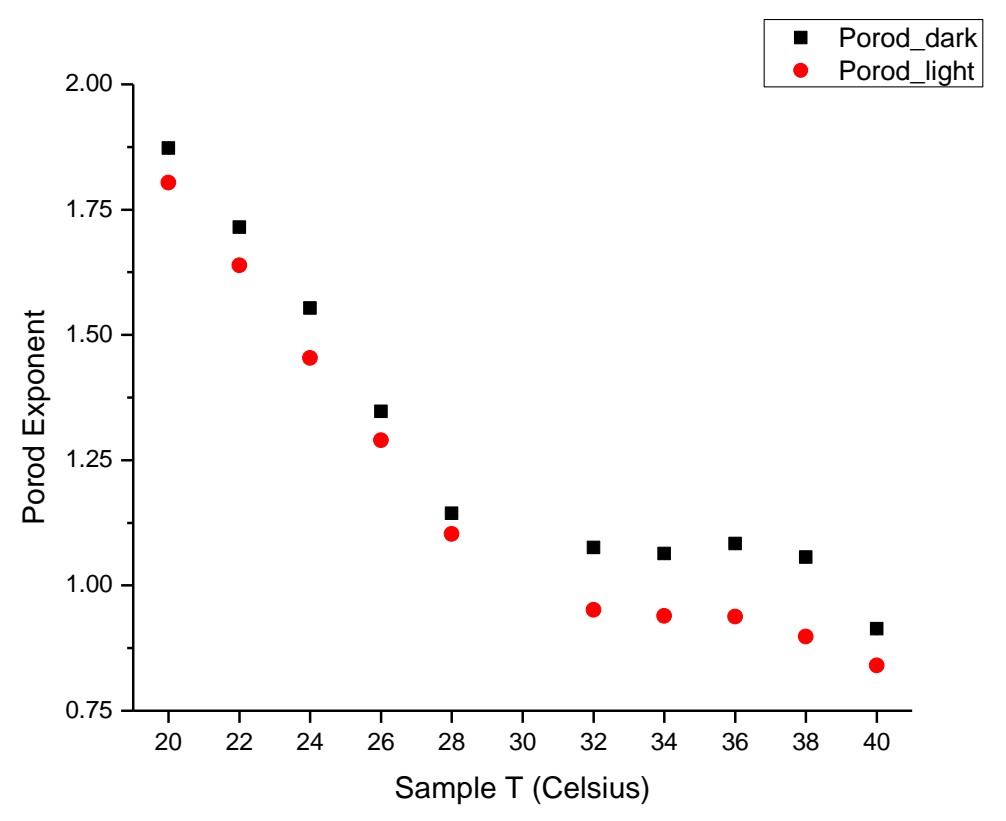

Figure 7: Porod exponent of free P3HT chains obtained via the excluded volume model, a measure of general chain conformation. These results indicate a slightly larger value, and thus more compact polymer chain, in the dark.

Though SANS analysis provides a great deal of insight into the nanometer level structure of the P3HT gelation process, in order to probe larger length scales of the full aggregates, expanding the Q range through USANS becomes necessary. Replicating exactly the experimental methodology of the above SANS setup, USANS measurements were conducted on an identical $17 \mathrm{wt} \% \mathrm{P} 3 \mathrm{HT}$ in $\mathrm{d}-\mathrm{ODCB}$ sample using the same temperature ramp protocol in the presence and absence of illumination. Example USANS data curves are plotted with their respective SANS curves from these experiments in Figure 8. 


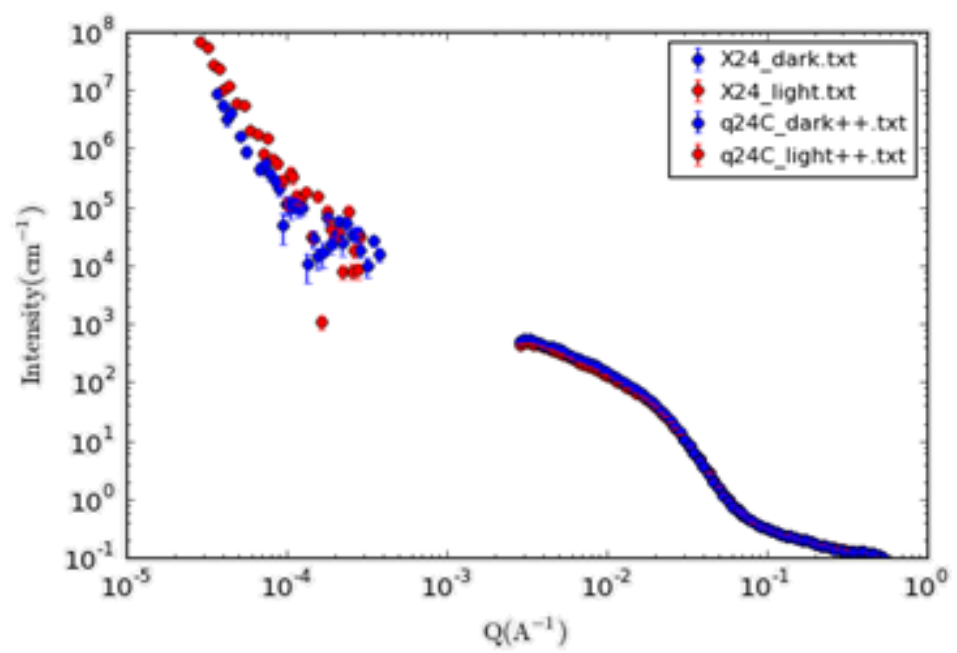

Figure 8: USANS and SANS curves of the $17 \mathrm{wt} \% \mathrm{P} 3 \mathrm{HT}$ solution in d-ODCB in both the light (red points) and dark (blue) at $24^{\circ} \mathrm{C}$.

Like the SANS data, the USANS curves show significant difference in absolute intensity between light and dark, a discrepancy which intensifies towards the end of gelation at lower temperatures. The data was fit using a Guinier-Porod Power law model, a general approach to determine the size and dimensionality of multi-scale scattering objects. ${ }^{32}$ Using this model, the radii of gyration of the large macro-aggregates were obtained, with the results shown in Figure 9. Intuitively, the overall aggregate size grows with decreasing temperature as increasingly more nanofibrils interact to form larger bundles as solvent quality decreases. Additionally, towards the end of gelation a very strong dependence of the $\mathrm{R}_{\mathrm{g}}$ on the illumination environment develops, indicating that light exposure influences the local structure enough to alter the larger structure of the gel as the gelation progresses. 


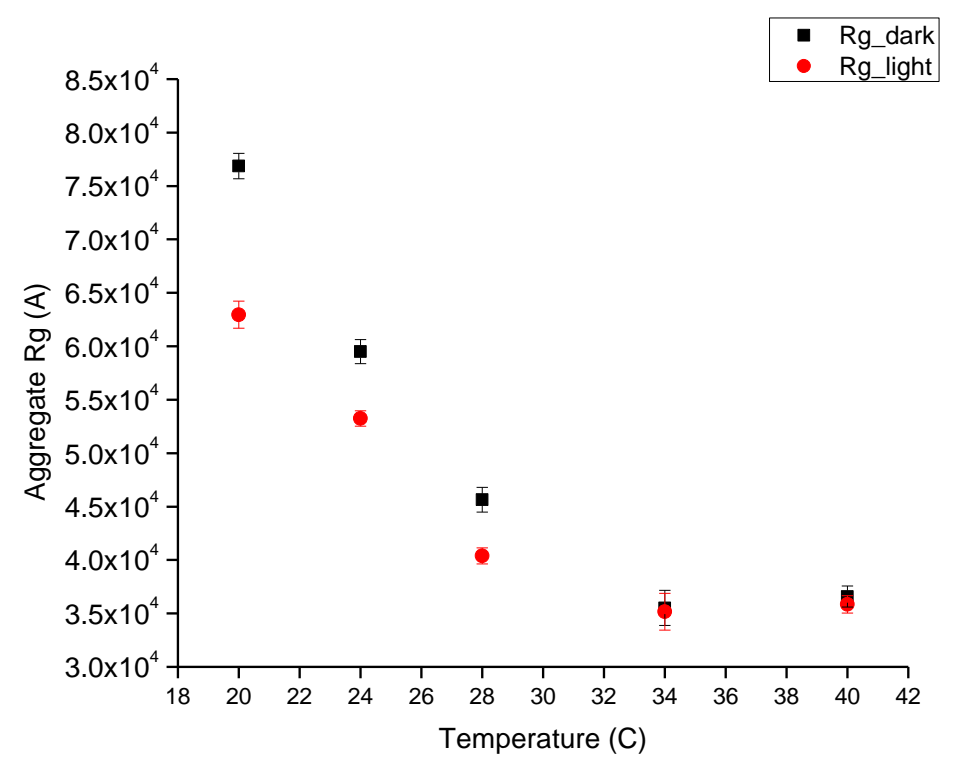

Figure 9: $\mathrm{R}_{\mathrm{g}}$ obtained from Guinier-Porod power law fit to USANS data for $17 \mathrm{wt} \% \mathrm{P} 3 \mathrm{HT}$ in dODCB. Black points indicate dark sample environment, red points illuminated environment.

\section{Discussion}

Analysis of the small and ultra small angle neutron scattering for a high concentration solution of an optoelectronic polymer offers special insight into the evolution of the structure of the forming gel as well as solution information during the gelling process. In particular, this analysis offers insight into the effect of white light illumination on the assembly of the conjugated polymers into the gel. Determining the size of the aggregated P3HT whiskers is crucial to understanding the structure-performance relationship in P3HT based active layers, as the size and shape of these P3HT semi-crystalline domains that emerge from these nanostructures has been shown to directly impact exciton dissociation and device efficiencies. $^{33,34}$ 
Analysis of SANS data monitors the cross-sectional surface area of the assembling fibrils. The data in Figure 4 shows fairly small changes in this cross-sectional area of the nanofibrils throughout gelation $(\sim 10 \%)$, indicating that once they are formed, the nanofibrils maintain a relatively uniform cross-sectional size and undergo further $\pi$-stacking to grow in length. Nanofibrils formed in the light show consistently smaller cross-sectional surface areas than their counterparts formed in the dark. This alteration in the assembly process is consistent with a change in the local chain structure or thermodynamic interaction between the polymer and solvent, ${ }^{25}$ which is initiated by light exposure and hinders aggregation. Figure 5 gives the scaling factor for the fit of the scattering data to the model, which is proportional to the amount of P3HT that has aggregated in solution. Intuitively, this increases with a decrease in temperature as decreasing solvent quality forces greater polymer-polymer interaction. Additionally, as in the case of nanofibril surface area, the "light" samples are much less aggregated than the samples that are formed in the dark, which is also consistent with a reduced extent of aggregation in the samples that are exposed to white light.

The combined ECM/PEV model also allows us to monitor the size and shape of the unaggregated P3HT chains. Figure 6 shows that decreasing temperature results a decrease in the $\mathrm{R}_{\mathrm{g}}$ of these free chains, a trend which becomes even more pronounced below $30^{\circ} \mathrm{C}$. This effect is what would be expected for a thermodynamically driven chain collapse due to decreasing solvent quality with decreasing temperature. The values for the free chain Porod exponent, shown in Figure 7, corroborate this model as well, as the exponent increases as gelation progresses, indicative of conversion from a semi-rigid rod conformation to a more compact state resembling a Gaussian coil. The impact of illumination is also present in the free chain characteristics, with white light illuminated samples boasting both larger radii of gyration and lower Porod exponents. 
Both parameters suggest that light exposure hinders the collapse of the free chains in solution, leading to a more expanded chain conformation that is less inclined to aggregate.

Figure 9 displays the analysis of the USANS data with a power law model documenting the $\mathrm{R}_{\mathrm{g}}$ of the aggregated P3HT bundles on the length scale of hundreds to thousands of nanometers. The sizes of these aggregates remain relatively constant through the higher temperatures, but as with the SANS data, these aggregates begin to grow as the temperature drops below $30^{\circ} \mathrm{C}$. Interestingly, the state of illumination dramatically impacts the final aggregate size, showing differences with illumination as aggregate growth accelerates below $30^{\circ} \mathrm{C}$. The non-illuminated samples produce significantly larger gelled macro-aggregates for all of these temperatures, culminating in a final aggregate radius that is over $20 \%$ larger than samples that are produced in the light. This trend is consistent with the SANS data, which showed that smaller nanofibrils are formed in samples under illumination.

Clearly illumination conditions have a profound effect on the aggregated structures of P3HT that are formed by a thermally driven gelation process. The presence of white light stunts the growth of P3HT nanofibril chains, which appears to be a direct result of the change in conformation of the individual free chains from which the nanofibril aggregates form. The free chains in the light-exposed P3HT solution are much less compact than identical solutions that are allowed to assemble in the dark. This larger radius of gyration appears to interfere with the required stacking that drives fibril formation. As a result, the cross-sectional area of the whisker aggregates that initially form remain distinctly smaller when the sample is illuminated. Predictably the macro-aggregate clusters that are composed of these smaller building blocks grow to smaller sizes than those that assemble in the dark. All structural parameters are 
consistent with this interpretation, including smaller $\mathrm{R}_{\mathrm{g}}$ values as determined by USANS and the volume fraction of aggregated P3HT chains elucidated via SANS.

Therefore, these results clearly demonstrate that the presence of incident light will dramatically affect the assembly and gelation of P3HT in concentrated solution. Moreover, illumination will also have a direct impact on the production of device active layers that utilize P3HT as the hole transport component, as the gelation process dictates the morphology and charge transport pathways in the P3HT in such devices. ${ }^{20,31}$ Therefore, because the morphologies that are generated through gel formation carry over to the final device architecture, the illumination conditions under which device fabrication occurs should not be overlooked. The results of this experimental program show that inconsistent illumination histories among otherwise identical samples can lead to large variations in size and amount of assembled gel structures. However, as this phenomenon is further studied and controlled it could also allow for protocols that offer pathways to targeted morphologies, including those that can be localized to areas of interest throughout a device by patterned illumination. Such an approach would provide a powerful in-situ means to impart hierarchical morphologies to the desired areas within active layers, while avoiding any damage or chemical alteration of the system in question.

\section{Conclusion}

Thermally driven gelation of the conjugated optoelectronic polymer P3HT has been monitored in-situ through a temperature ramp with both small and ultra small angle neutron scattering to monitor the change in the assembly process due to the presence or absence of illumination. Data analysis clearly indicates formation of cylindrical nanofibril aggregates which 
themselves further self-assemble into macro-aggregates, a process that occurs quickly with decreasing temperature. Careful analysis of the resultant scattering patterns demonstrates that the presence of illumination on the gelling solution results in a decrease in the driving force for gelation, smaller nanofibrils, and a lower volume fraction of gelled P3HT. This behavior is obvious across multiple length scales and leads to ultimately smaller P3HT microstructures than the non-illuminated counterparts. These results, therefore, indicate for the first time that careful control of the illumination conditions during P3HT active layer based device fabrication is crucial to maintain reliability and reproducibility in their production and performance. Additionally, while failure to effectively monitor and control illumination environment during device fabrication may ultimately yield undesirable and inconsistent architectures, morphologies and performance, proper harnessing of these optoelectronic structural effects through carefully controlled illumination methodologies could conversely open the door to a new field of localized morphology control and active layer structural manipulation geared towards further optimizing organic electronic device performance.

\section{Acknowledgements:}

The authors gratefully acknowledge the National Science Foundation (DMR-1409034) for support of this project. We also acknowledge the support of the National Institute of Standards and Technology, U.S. Department of Commerce, in providing the USANS facilities used in this work, where these facilities are supported in part by the National Science Foundation under Agreement No. DMR-0944772. The SANS experiments of this research was completed at ORNL's High Flux Isotope Reactor, which was sponsored by the Scientific User Facilities Division, Office of Basic Energy Sciences, US Department of Energy. 
Keywords: optically active conjugated polymers; polymer gels; neutron scattering

\section{References}

(1) Brinkmann, M. Structure and Morphology Control in Thin Films of Regioregular poly(3Hexylthiophene). J. Polym. Sci. Part B Polym. Phys. 2011, 49, 1218-1233.

(2) Wang, H.; Xu, Y.; Yu, X.; Xing, R.; Liu, J.; Han, Y. Structure and Morphology Control in Thin Films of Conjugated Polymers for an Improved Charge Transport. Polymers (Basel). 2013, 5 (4), 1272-1324.

(3) Chang, M.; Lee, J.; Kleinhenz, N.; Fu, B.; Reichmanis, E. Photoinduced Anisotropic Supramolecular Assembly and Enhanced Charge Transport of Poly(3-Hexylthiophene) Thin Films. Adv. Funct. Mater. 2014, 24 (28), 4457-4465.

(4) Yu, J.; Hu, D.; Barbara, P. Unmasking Electronic Energy Transfer of Conjugated Polymers by Suppression of O2 Quenching. Science (80-. ). 2000, 289, 1327-1330.

(5) Adhikari, N.; Qiao, Q. Device and Morphological Engineering of Organic Solar Cells for Enhanced Charge Transport and Photovoltaic Performance. J. Photonics Energy 2015, 5, 57207, 1-17.

(6) Yang, X.; Loos, J.; Veenstra, S. C.; Verhees, W. J. H.; Wienk, M. M.; Kroon, J. M.; Michels, M. a J.; Janssen, R. a J. Nanoscale Morphology of High-Performance Polymer Solar Cells. Nano Lett. 2005, 5 (4), 579-583.

(7) Huynh, W. U.; Dittmer, J. J.; Alivisatos, A. P. Hybrid Nanorod-Polymer Solar Cells. Science (80-. ). 2002, 295, 2425-2428.

(8) Schwartz, B. J. Conjugated Polymers as Molecular Materials: How Chain Conformation and Film Morphology Influence Energy Transfer and Interchain Interactions. Annu. Rev. Phys. Chem. 2003, 54, 141-172.

(9) Xu, W.; Tang, H.; Lv, H.; Li, J.; Zhao, X.; Li, H.; Wang, N.; Yang, X. Sol-gel Transition of poly(3-Hexylthiophene) Revealed by Capillary Measurements: Phase Behaviors, Gelation Kinetics and the Formation Mechanism. Soft Matter 2012, 8, 726.

(10) Koppe, M.; Brabec, C. J.; Heiml, S.; Schausberger, A.; Duffy, W.; Heeney, M.; McCulloch, I. Influence of Molecular Weight Distribution on the Gelation of P3HT and Its Impact on the Photovoltaic Performance. Macromolecules 2009, 42, 4661-4666.

(11) Guo, R. H.; Hsu, C. H.; Hua, C. C.; Chen, S. a. Colloidal Aggregate and Gel Incubated by Amorphous Conjugated Polymer in Hybrid-Solvent Medium. J. Phys. Chem. B 2015, 119 (7), 3320-3331.

(12) Alcazar, D.; Wang, F.; Swager, T. M.; Thomas, E. L. Gel Processing for Highly Oriented Conjugated Polymer Films. Macromolecules 2008, 41 (24), 9863-9868.

(13) Yang, C. Y.; Heeger, a. J.; Cao, Y. Microstructure of Gel-Processed Blends of Conjugated Polymer and Ultrahigh Molecular Weight Polyethylene. Polymer (Guildf). 2000, 41, 4113-4118. 
(14) Lee, C. S.; Yin, W.; Holt, A. P.; Sangoro, J. R.; Sokolov, A. P.; Dadmun, M. D. Rapid and Facile Formation of P3HT Organogels via Spin Coating: Tuning Functional Properties of Organic Electronic Thin Films. Adv. Funct. Mater. 2015, 25 (36), 5848-5857.

(15) Wicklein, A.; Ghosh, S.; Sommer, M.; Würthner, F.; Thelakkat, M. Self-Assembly of Semiconductor Organogelator Nanowires for Photoinduced Charge Separation. ACS Nano 2009, 3 (5), 1107-1114.

(16) Terech, P.; Weiss, R. G. Low Molecular Mass Gelators of Organic Liquids and the Properties of Their Gels. 1997, 2665 (97).

(17) Chang, J. F.; Sun, B.; Breiby, D. W.; Nielsen, M. M.; Sölling, T. I.; Giles, M.; McCulloch, I.; Sirringhaus, H. Enhanced Mobility of poly(3-Hexylthiophene) Transistors by SpinCoating from High-Boiling-Point Solvents. Chem. Mater. 2004, 16 (23), 4772-4776.

(18) Herrmann, H.; Landau, D.; Stauffer, D. New Universality Class for Kinetic Gelation. Phys. Rev. Lett. 1982, 49 (6), 412-415.

(19) Zaccarelli, E. Colloidal Gels: Equilibrium and Non-Equilibrium Routes. J. Phys. Condens. Matter 2007, 19, 323101, 1-50.

(20) Newbloom, G. M.; Kim, F. S.; Jenekhe, S. a.; Pozzo, D. C. Mesoscale Morphology and Charge Transport in Colloidal Networks of Poly(3-Hexylthiophene). Macromolecules 2011, 44, 3801-3809.

(21) Tretiak, S.; Saxena, A.; Martin, R.; Bishop, A. Conformational Dynamics of Photoexcited Conjugated Molecules. Phys. Rev. Lett. 2002, 89 (9), 097402.

(22) Botiz, I.; Freyberg, P.; Stingelin, N.; Yang, A. C.-M.; Reiter, G. Reversibly Slowing Dewetting of Conjugated Polymers by Light. Macromolecules 2013, 46, 2352-2356.

(23) Botiz, I.; Freyberg, P.; Leordean, C.; Gabudean, A.; Astilean, S.; Yang, A. C.; Stingelin, N. Enhancing the Photoluminescence Emission of Conjugated MEH-PPV by Light Processing. ACS Appl. Mater. Interfaces 2014, 6 (7), 4974-4979.

(24) Kumar, J.; Li, L.; Jiang, X.; Kim, D.; Lee, T.; Tripathy, S. Gradient Force: The Mechanism for Surface Relief Grating Formation in Azobenzene Functionalized Polymers. Appl. Phys. Lett. 1998, 72 (17), 2096.

(25) Morgan, B.; Dadmun, M. D. Illumination of Conjugated Polymer in Solution Alters Its Conformation and Thermodynamics. Macromolecules 2016, 49 (9), 3490-3496.

(26) https://www.ncnr.nist.gov/programs/sans/data/red_anal.html (accessed Feb 1, 2013).

(27) Barker, J. G.; Glinka, C. J.; Moyer, J. J.; Kim, M. H.; Drews, A. R.; Agamalian, M. Design and Performance of a Thermal-Neutron Double-Crystal Diffractometer for USANS at NIST. J. Appl. Crystallogr. 2005, 38 (6), 1004-1011.

(28) Schulz, G. Z. Phys. Chem. 1935, 43, 25.

(29) Mittelbach; Porod. Acta Phys. Austriaca 1961, 14, 185-211.

(30) Yin, W.; Dadmun, M. A New Model for the Morphology of P3HT / PCBM Organic Photovoltaics from Small-Angle Neutron Scattering : Rivers and Streams. ACS Nano 
2011, 5 (6), 4756-4768.

(31) Newbloom, G. M.; Weigandt, K. M.; Pozzo, D. C. Electrical, Mechanical, and Structural Characterization of Self-Assembly in poly(3-Hexylthiophene) Organogel Networks. Macromolecules 2012, 45, 3452-3462.

(32) Guinier, A.; Fournet, G. Small-Angle Scattering of X-Rays; Wiley: New York, 1955.

(33) Park, M. S.; Aiyar, A.; Park, J. O.; Reichmanis, E.; Srinivasarao, M. Drain Current in Poly(3-Hexylthiophene) Solutions during Film Formation: Correlations to Structural Changes. ChemNanoMat 2015, 1 (1), 32-38.

(34) Xiao, G.; Guo, Y.; Lin, Y.; Ma, X.; Su, Z.; Wang, Q. Controlled Evaporative SelfAssembly of Poly(3-Hexylthiophene) Monitored with Confocal Polarized Raman Spectroscopy. Phys. Chem. Chem. Phys. 2012, 14, 16286-16293. 